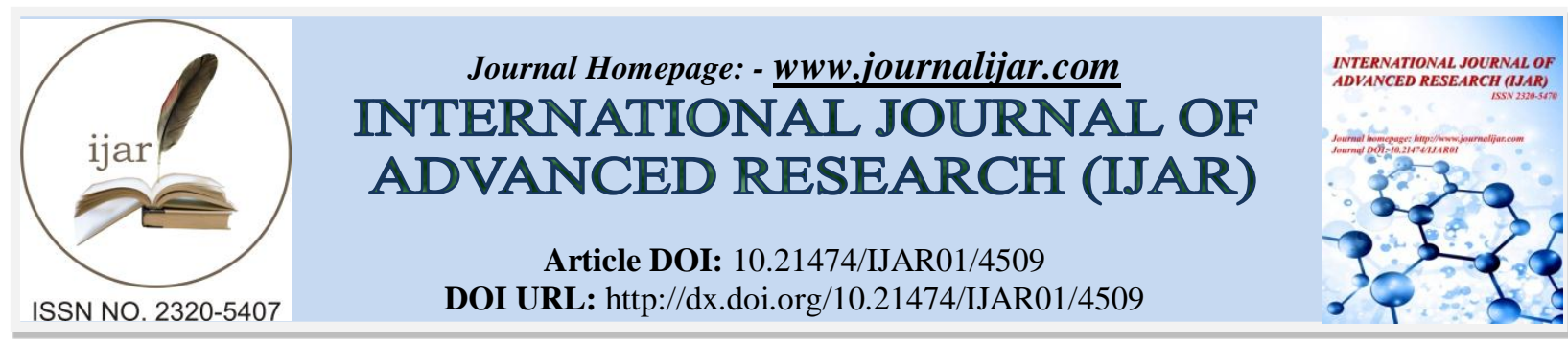

RESEARCH ARTICLE

\title{
NEPRILYSIN LIKE PEPTIDES AND SMALL MOLECULES IN THE COMBAT AGAINST AMYLOID BETA PLAQUES OF ALZHEIMER'S DISEASE THROUGH IN-SILICO BINDING STUDIES.
}

\section{${ }^{*}$ Uma Anusha Nukala ${ }^{1}$ and Mrs. P. Sahithi ${ }^{2}$.}

1. M.Tech (Biochemical Engineering \& Biotechnology), University College of Technology, Osmania University, Hyderabad, India.

2. Former Assistant professor, University College of Technology, Osmania University, Hyderabad, India.

\section{Manuscript Info}

Manuscript History

Received: 17 April 2017

Final Accepted: 19 May 2017

Published: June 2017

Key words:-

Alzheimer's disease, Amyloid Beta, Cognitive abilities, Neprilysin, Senile Plaques

\section{Abstract}

Alzheimer's Disease, being one of the most detrimental Neurodegenerative diseases, is an irreversible, progressive brain disorder with changes in nerve cells resulting in their death and furthermore leading to the loss of intellectual and cognitive abilities. Despite the commercial availability of few drugs, Alzheimer's disease is the sixth leading causes of death globally and the major public health concern, owing to a constraining coercion for action against it. For the same, many drug targets were identified among which the most potential one is the amyloid beta protein which contributes to the pathological lesions of Alzheimer's disease, the Amyloid Beta Plaques or Senile Plaques. In the present study, two approaches - one approach plying Neprilysin like peptides and the other approach employing a set of Small molecules, were used to target these pathological lesions with the help of Bioinformatics tools such as Discovery Studio and designed therapeutic molecules that can potentially combat against these plaques and reduce their content in neurons which might result in an improved communication and thereby enhancing the condition of the diseased person.

Copy Right, IJAR, 2017,. All rights reserved.

\section{Introduction:-}

Of all the devastating infirmities prevailing the world contemporaneously, brain related disorders have been proven to be an enormous disease burden in terms of human suffering and economic cost. Peculiarly brain disorders termed as "Neurodegenerative diseases/disorders (NDDs)" that involve the progressive loss of structure or function of neurons, including death of neurons, are the most fatal ones. One among such deleterious NDDs is the Alzheimer's disease which is an irreversible, progressive brain disorder related to alterations in nerve cells that result in their death and furthermore leading to the loss of intellectual and cognitive abilities, such as thinking, remembering and reasoning, that is excruciating enough to interfere with daily functioning.

Alzheimer's disease being the sixth leading cause of death with as high a mortality rate as 1 in every 3 seniors dies, the prevalence and incidence projections denote that the number of people with Alzheimer disease will perpetuate to grow, categorically among the oldest old, and countries in demographic transition will experience the greatest magnification. The total number of people with AD ecumenical in 2010 is estimated at 35.6 million and is projected to proximately double every 20 years, to 65.7 million in 2030 and 115.4 million in 2050. Much of the 
incrementation will be in developing countries, with the most rapid magnification in the elderly population taking place in China, India, and their south Asian and western Pacific neighbours. In 2010, Europe had an estimated 10 million disease cases and predicated on Amalgamated Nation's demographic forecast this figure will elevate to 14 million in 2030. It is projected that by 2050, people aged 60 and over will account for $22 \%$ of the world's population with four-fifths living in Asia, Latin America or Africa. Visually examining these data, it is ostensible that there is an imperative duress for action owing to the fact that the Alzheimer's disease has become a major public health concern as the world's population ages. For the same, many drug targets were identified among which one of the most potential one is the Amyloid Beta protein that is biosynthesized from the amyloid precursor protein (APP) by the operation of the $\beta$ - and $\gamma$ - secretases.

The aggregation of this Amyloid Beta protein into amyloid plaques/fibrils is a major pathological hallmark of the Alzheimer's disease, evidently implying that $A \beta$ aggregation is critical for neurodegeneration, propounding that hindering this process may be an effective therapeutic approach for the treatment of AD. A number of small molecules have been reported to inhibit $\mathrm{A} \beta$ fibrillogenesis among which the following small molecules or analogues of small molecules reported previously to bind amyloid or to tamper with protein aggregation and/or toxicity or scrutinized for such activities were taken for performing protein - ligand docking studies with $\mathrm{A} \beta$ for Objective -1 and to obtain the best ligand molecule: Apigenin, Azure C, Basicblue41, (Trans,Trans)-1-Bromo-2,5-Bis(3Hydroxycarbonyl-4-Hydroxy) Styrylbenzene (BSB), Chicago Sky Blue 6B, Congored, B -Cyclodextrin, Curcumin, Daunomycin Hydrochloride, Dimethyl Yellow, Direct Red 80, 2,2'Dihydroxybenzophenone, Hexadecyltrimethylammonium Bromide (C16), Hemin Chloride, Hematin, Indomethacin, Juglone, Lacmoid, Meclocycline Sulfosalicylate, Melatonin, Myricetin, 1,2-Naphthoquinone, Nordihydroguaiaretic Acid, R(-)Norapomorphine Hydrobromide, Orangeg, OVanillin (2-Hydroxy-3-Methoxybenzaldehyde), Pherphenazine, Phthalocyanine, Rifamycinsv, Phenol Red, Rolitetracycline, Quinacrine Mustard Dihydrochloride, Thioflavin S, Tht, and Trimethyl (Tetradecyl) Ammonium Bromide (C17). In addition, Diallyl Tartar, Eosin Y, Fenofibrate, Neocuproine, Nystalin, Octadecyl Sulfate, and Rhodamine B have also been tested.

As an additional novel approach to the Alzheimer's disease therapy, the focus of the therapeutic strategies of Objective -2 has been aimed at fostering the $A \beta$ degradation that aids in the maintenance of the steady-state level of amyloid $\beta$-peptide $(A \beta)$ by promoting the catabolism of $A \beta$ by a variety of proteolytic enzymes, thereby balancing the A $\beta$ biosynthesis from APP. For the same, as a matter of paramount importance, this study has focussed on Neprilysin (NEP), family of zinc metalloproteinases, as it is known to play a major role in the degradation of both $\mathrm{A} \beta_{1-40}$ and $A \beta_{1-42}$ in vitro and in vivo and also in terminating neuropeptide signalling aiding the brain functioning, while it's decrease during ageing or after pathologies such as hypoxia or ischemia contribute significantly to the development of AD pathology. On the grounds of Structure Activity Relationships (SAR) which states that similar structures possess similar biological activities / functionalities, this task has been consummated by taking / constructing peptide molecules with the following 10 sequences similar to Neprilysin in order to perform protein peptide docking studies and identify the best peptide molecule: A4TA86; B1MB62; ELNFTPNWGT (pepbank); HFPGP (pepbank); MEHFPGP (pepbank); P24559; Q03R34; Q18G54; TKPRP (pepbank); TKPRPGP (pepbank)

Although, there are a few drugs commercially available so far, these drugs are not devoid of their side effects. Hence, this in-silico study is an attempt to detect more physiologically suitable and compatible therapeutic molecules with least possible side effects.

\section{Materials and Methodology:- \\ Materials:- \\ Servers:-}

The online servers / databases used to consummate this study were Uniprot (http://www.uniprot.org/), RAMPAGE Server (http://mordred.bioc.cam.ac.uk/ rapper/rampage.php), Protein Data Bank (http://www.rcsb.org/), Fold Amyloid Server (http://antares.protres.ru/fold-amyloid/), Pepbank Server (http://pepbank.mgh.harvard.edu/) and Pubchem (https://pubchem.ncbi.nlm.nih.gov).

\section{Tools \& Software:-}

The online similarity search \& sequence analysis tool BLAST (www.ncbi.nlm.nih.gov/blast) and the Accelrys Discovery Studio 2.5 software have been employed to carry out the objectives of the study to completion. 


\section{Methodology:-}

Target Protein Structure Preparation:-

With the target protein being Amyloid Beta $(\mathrm{A} \beta)$ for both Objective - 1 \& Objective - 2, the Uniprot is searched for all the available Amyloid Beta human $(\mathrm{A} \beta$ ) structures of which structures of the $\mathrm{A} \beta$ human protein with Accession number: P05067 (A4_HUMAN) have been submitted to RAMPAGE server for Ramachandran Plot Analysis that yielded the best protein structure bearing PDB ID: "IIYT" (Figure 1), which was downloaded from PDB for protein preparation.

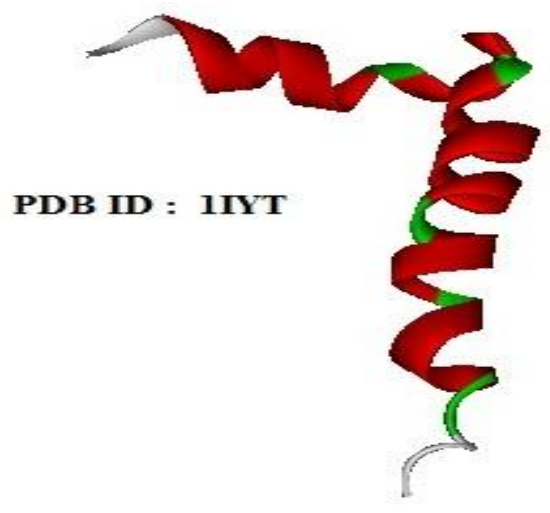

Figure 1:- 3D - structure of the selected amyloid beta protein structure with PDB ID: " $1 I Y T$ "

Thus, the obtained A $\beta$ protein structure is loaded into the 3D-Window of Discovery Studio 2.5 and then submitted to protein modelling by enabling the parameters viz., Incomplete residue, Atom order, Add hydrogens, Valency, \& Charge, which is then subjected to energy minimization to obtain a stable configuration by applying "Forcefield" and "Simulation" with the aid of "Steepest Descent" and "Conjugate Gradient" minimization algorithms which are repeated until the minimization criteria shows a "Gradient Tolerance Satisfied" message indicating that the protein has reached a stable configuration which is then saved in '.mol' format (Figure 2).

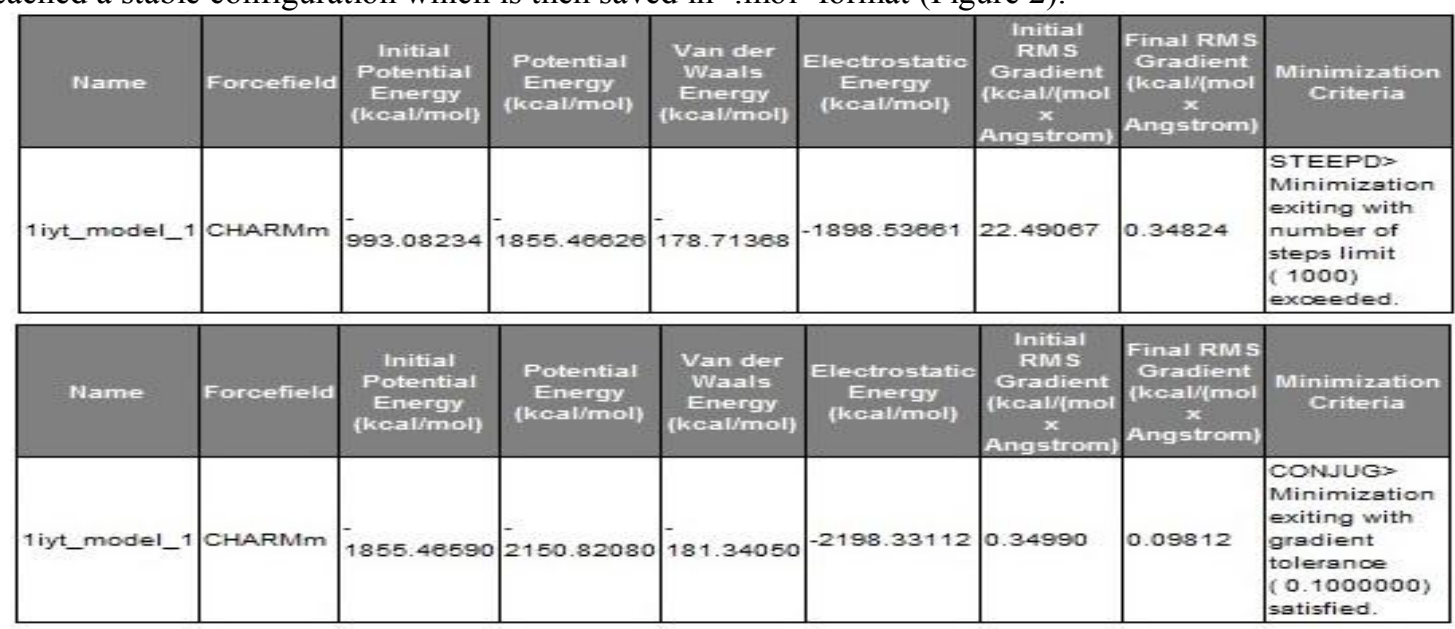

Figure 2:- Summary of Steepest descent \& Conjugate gradient energy minimization algorithms

\section{Identification of Amyloidogenic regions and Active site sphere construction:-}

The positions of Amyloidogenic regions in polypeptide chains which are responsible for amyloid formation and aggregation were predicted to be at two sites namely: $16-21$ amino acid region and $32-36$ amino acid region, with the aid of Fold Amyloid Server by loading the FASTA sequence of prepared protein and selecting "Predict". Followed by defining the "Active Site Spheres" around the amyloidogenic regions of the minimized A $\beta$ protein structure by employing the Receptor - ligand interactions tool in the Discovery Studio (Figure 3). 


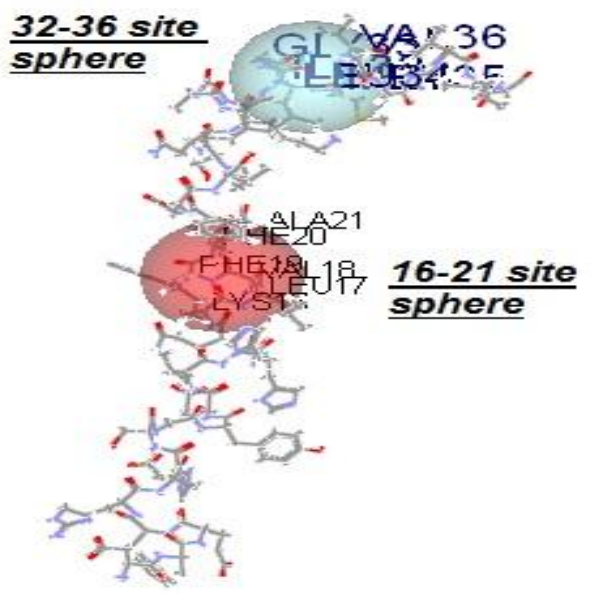

Figure 3:- Active site spheres around both the identified amyloidogenic regions of the final minimized A $\beta$ protein structure

\section{Compounds that inhibit A $\beta$ protein aggregation (Objective 1):- \\ Ligand Preparation:-}

The small molecules (Figure 4) which were found to have an inhibitory effect on the A $\beta$ protein aggregation were downloaded from Pubchem and were then subjected to Ligand preparation using Discovery Studio, by employing Receptor - Ligand interactions tool and selecting "Prepare Ligands" with "Lipinski filter" selection criteria as "True".
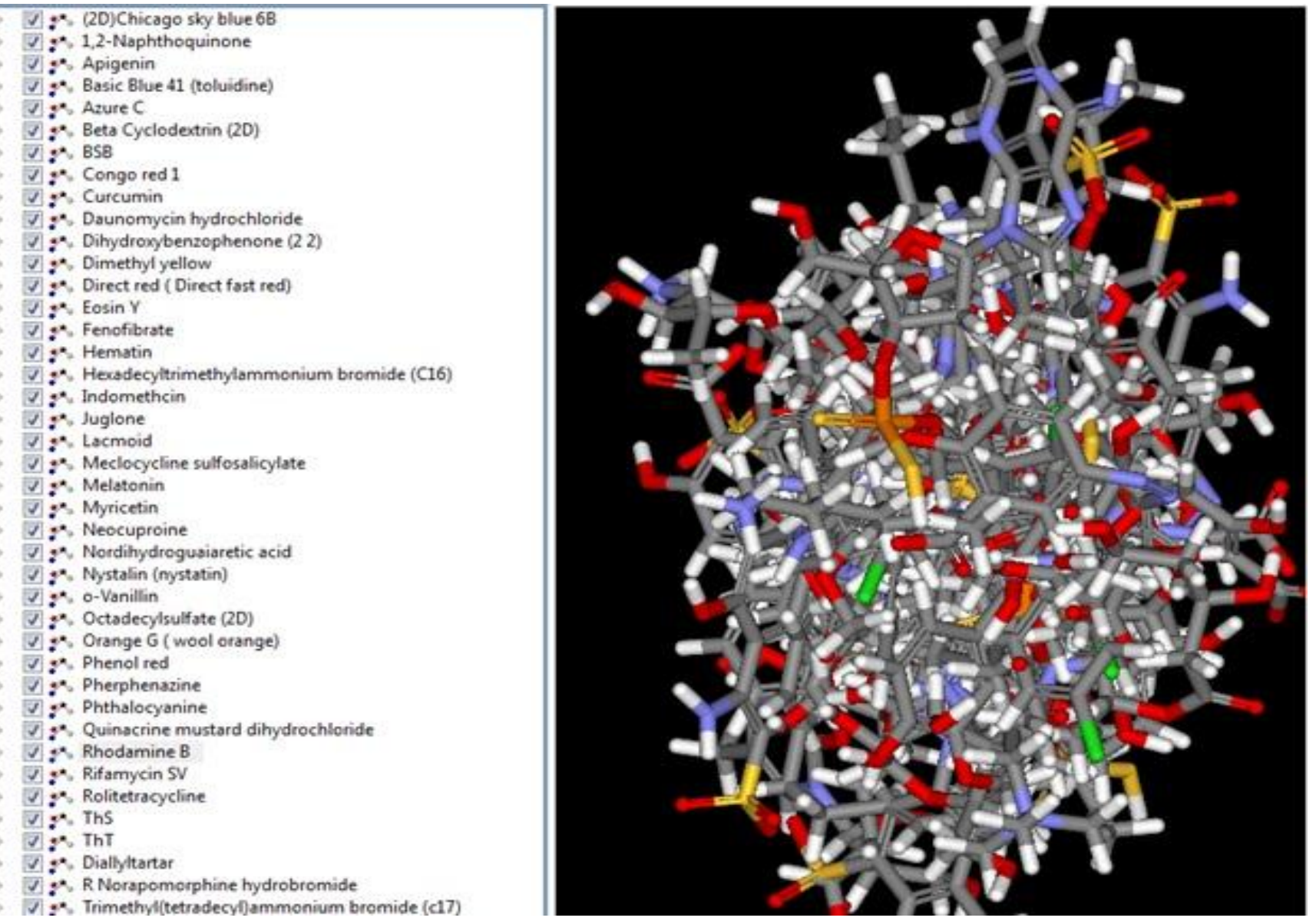

Figure 4:- Small Molecules (Left) \& Prepared ligands (Right) 


\section{Docking by Libdock algorithm:-}

Following the preparation of Ligands from the selected small molecules, the Docking studies were consummated in Discovery Studio, with the aid of Libdock algorithm, by taking the Minimized A $\beta$ protein structure (PDB ID: "1IYT") as the Input receptor, the prepared ligands as the Input Ligands and the active site spheres defined around the amyloidogenic regions viz., $16-21$ amino acid region \& $32-36$ amino acid region as the Input Site Spheres.

Neprilysin like peptide molecules for Aß peptide catabolism (Objective - 2):-

Identification of Neprilysin like peptide sequences:-

With the help of Protein BLAST and Pepbank Server, the following peptide sequences similar to Neprilysin, as listed in Table 1, were identified and selected for further analysis. The fasta sequence of the Neprilsyin Human protein structure with $P D B I D$ : "IDMT", that was selected by Ramachandran Plot Analysis through RAMPAGE server, was employed for running the Protein BLAST and the sequences with highest identity $\%$ were selected for further analysis. While the Pepbank Server, peptide database, was ransacked for Neprilysin like sequences and the sequences with high interaction score were selected for further analysis.

Table 1:- Neprilysin like peptide sequences identified

\begin{tabular}{|c|c|c|c|c|c|}
\hline \multicolumn{2}{|c|}{ BLAST PEPTIDE SEQUENCES } & \multicolumn{3}{c|}{ PEPBANK SERVER PEPTIDE SEQUENCES } \\
\hline Name & Sequence & Identity & Name & Sequence & Score \\
\hline P24559 & RFRVNAF & $100 \%$ & HFPGP & HFPGP & 0.69 \\
\hline Q03R34 & NSFIVHEH & $88 \%$ & MEHFPGP & MEHFPGP & 0.69 \\
\hline A4TA86 & EHEWVQR & $86 \%$ & TKPRP & TKPRP & 0.69 \\
\hline B1MB62 & EHEWVRR & $86 \%$ & TKPRPGP & TKPRPGP & 0.69 \\
\hline Q18G54 & FIVHEH & $100 \%$ & ELNFTPNWGT & ELNFTPNWGT & 0.5 \\
\hline
\end{tabular}

Construction \& Minimization of peptide molecules:-

The selected peptide sequences were then employed in the construction of peptide molecules for further studies to be performed with the aid of Protein Modelling tool in Discovery Studio by selecting Build and Edit protein with the Conformation as "Left-hand alpha helix" as the protein structure originally seems to have a left-hand alpha helix conformation and at Choose Amino acid the peptide sequences that have been selected for study were pasted individually to generate the respective peptide molecules. Thus, constructed peptide molecules were then subjected to Dreiding minimize for approximately 20 times until there is no significant change in the energy of the molecule and then to Forcefield: CharmM followed by the Simulation through Minimization by employing the Smart minimizer algorithm with maximum steps of 500 .

\section{Docking by Z-Dock \& R-Dock Algorithms:-}

The minimized A $\beta$ protein structure (PDB ID: "IIYT") and the minimized peptide molecules were then subjected to protein - peptide docking process through Discovery studio with the help of two different algorithms namely Z-dock and R- dock. With the Input Receptor being Minimized A protein structure (PDB ID: "IIYT") and the Input Ligands being Peptide Molecule, the Z-Dock was performed for each of the peptide molecule individually with the Clustering criteria set to Angular step size of 6, Top poses of 2000, RMSD of 6.0, Interface of 9.0 and a Maximum number of clusters being 30, followed by the Simulation through CHARMm Polar H in the Forcefield. Then the RDock was performed for all the peptide molecules with the Input Receptor being Z-Dock results protein "IIYT", Input Ligands being Z-Dock results Peptide Molecule and the Input poses being all the 30 clusters individually.

\section{Results:-}

\section{Libdock Docking Results for Objective 1:-}

The protein - ligand docking studies consummated with the aid of Libdock algorithm, with the " 805 " number of prepared ligands taken as the Input Ligands, the Minimized A $\beta$ protein structure (PDB ID: "1IYT") as the Input receptor and the active site spheres defined around the amyloidogenic regions viz., $16-21$ amino acid region $\& 32$ - 36 amino acid region as the Input Site Spheres, yielded results stating that the $16-21$ amino acid region had no ligands docked with 196 failed poses while the $32-36$ amino acid region had 381 successful docked poses with 167 failed poses (Figure 5). 


\section{5 input ligands \\ (a) \\ 17714 conformers generated \\ 196 poses failed to dook \\ (b) \\ 805 input ligands \\ 17714 conformers generated \\ 167 poses failed to dook \\ no ligands doded \\ 381 poses dodked}

Figure 5:- (a) Docking results summary of 16-21 active site sphere

(b) Docking results summary of $32-36$ active site sphere

Since the docking results of the active site $16-21$ amino acid region showed that there were no ligands docked, the complete docking scores of the all small molecule compounds docked along with the Interacting amino acids for the active site 32 - 36 amino acid region were tabulated in the following Table 2 that yielded in the best Ligand molecule as Octadecylsulfate with highest libdock score as "84.927" (Figure 6).

Table 2:- Libdock scores of the docking results of $32-36$ active site region

\begin{tabular}{|c|c|c|}
\hline COMPOUND NAME & LIBDOCK SCORE & INTERACTING AMINO ACIDS \\
\hline Chicago sky blue 6B & 17.588 & LYS28, ILE31, LEU34, MET35 \\
\hline Apigenin & 31.984 & MET35, LYS28, ILE32 \\
\hline Azure C & 24.577 & LYS28, MET35, ILE32, ILE31 \\
\hline Curcumin & 62.827 & VAL24, ILE31, ILE32, MET35, LYS28 \\
\hline Daunomycin hydrochloride & 24.394 & MET35, VAL39, LYS28 \\
\hline Hematin & 70.066 & VAL24, LYS28, ILE31, ILE32, MET35, VAL36, \\
\hline $\begin{array}{c}\text { Hexadecyltrimethylammonium } \\
\text { bromide (C16) }\end{array}$ & 75.194 & LYS28, MET35, VAL36 \\
\hline Indomethcin & 66.362 & ILE31, LYS28, MET35 \\
\hline Myricetin & 31.769 & LYS28, MET35 \\
\hline Octadecylsulfate & 84.927 & LYS28, ILE31, ILE32, MET35, VAL36, VAL39 \\
\hline Phenol red & 35.954 & LYS28, ILE31, ILE32, MET35, VAL39 \\
\hline Pherphenazine & 63.713 & LYS28, ILE31, ILE32, MET35 \\
\hline $\begin{array}{c}\text { Quinacrine mustard } \\
\text { dihydrochloride }\end{array}$ & 70.293 & LYS28, ILE31, ILE32, MET35, VAL36, VAL39 \\
\hline Diallyltartar & 56.138 & LYS28, MET35, ILE32 \\
\hline $\begin{array}{c}\text { Trimethyl(tetradecyl)ammonium } \\
\text { bromide (c17) }\end{array}$ & 64.478 & \\
\hline Lacmoid & 30.594 & \\
\hline
\end{tabular}

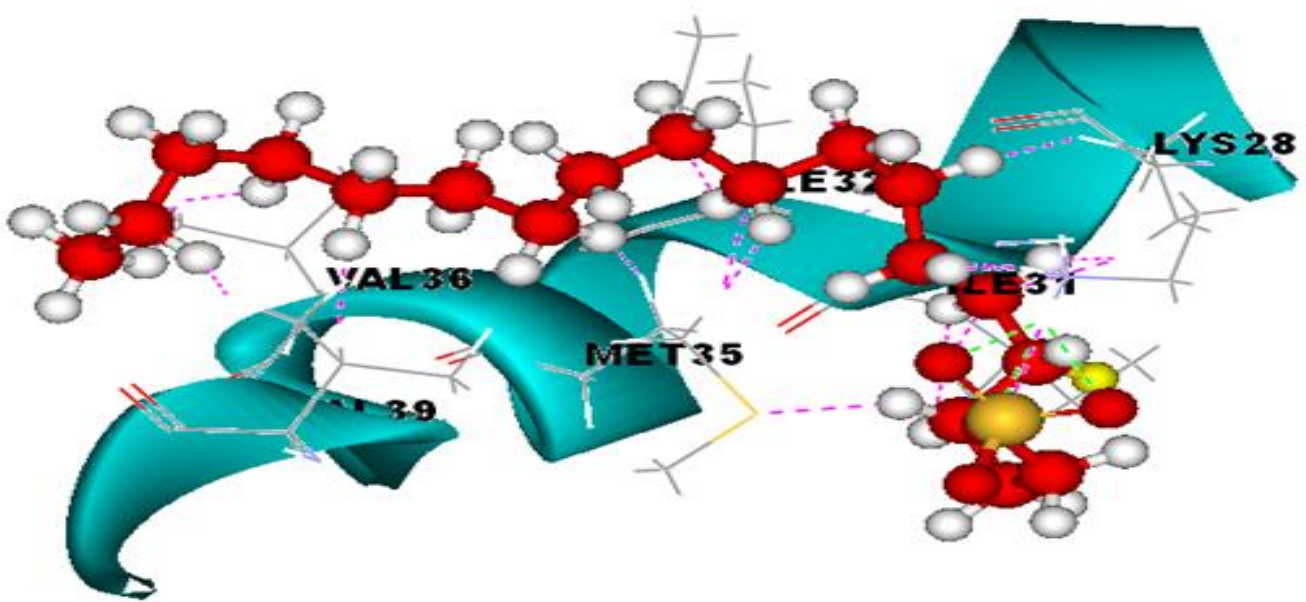

Figure 6:- Octadecylsulfate with highest libdock score as 84.927 
Z-Dock \& R-Dock Docking Results for Objective 2:-

The results of the protein - peptide docking studies consummated with the aid of Z-Dock algorithm followed by the R-Dock algorithm for the selected \& constructed 10 peptide molecules have been tabulated below in Table 3 with their respective poses having highest Z-Dock score and the cluster with the (-ve) highest R-Dock score.

Table 3:- Z-Dock and R-Dock scores of the peptide sequences

\begin{tabular}{|c|c|c|}
\hline PEPTIDE SEQUENCE & $\begin{array}{c}\text { Z-DOCK SCORE } \\
\text { (HIGHEST) }\end{array}$ & CLUSTER WITH (-VE) HIGHEST R-DOCK SCORE \\
\hline A4TA86 & 10.28 for Pose1159 & Cluster 17 with -7.074665 score \\
\hline B1MB62 & 9.76 for Pose514 & Cluster 15 with -16.01563 score \\
\hline ELNFTPNWGT (pepbank) & $\mathbf{1 0 . 3 4}$ for Pose168 & Cluster 26 with -19.663977 score \\
\hline HFPGP (pepbank) & 8.96 for Pose1278 & Cluster 16 with -13.74314 score \\
\hline MEHFPGP (pepbank) & 9.56 for Pose1162 & Cluster 2 with -17.936031 score \\
\hline P24559 & 8.54 for Pose744 & Cluster 16 with -13.908278 score \\
\hline Q03R34 & 8.4 for Pose735 & Cluster 6 with -12.120217 score \\
\hline Q18G54 & 8.08 for Pose428 & Cluster 26 with -12.728835 score \\
\hline TKPRP (pepbank) & 8.34 for Pose460 & Cluster 1 with -14.91993 score \\
\hline TKPRPGP (pepbank) & 9.48 for Pose1244 & Cluster 2 with -17.27739 score \\
\hline
\end{tabular}

These results have yielded in the best peptide molecule sequenced ELNFTPNWGT, which was selected from pepbank server, that had the highest Z-Dock Score of 10.34 for Pose168 (Figure 7) with the Cluster 26 showing the (-ve) highest R-Dock score as -19.663977 (Figure 8).

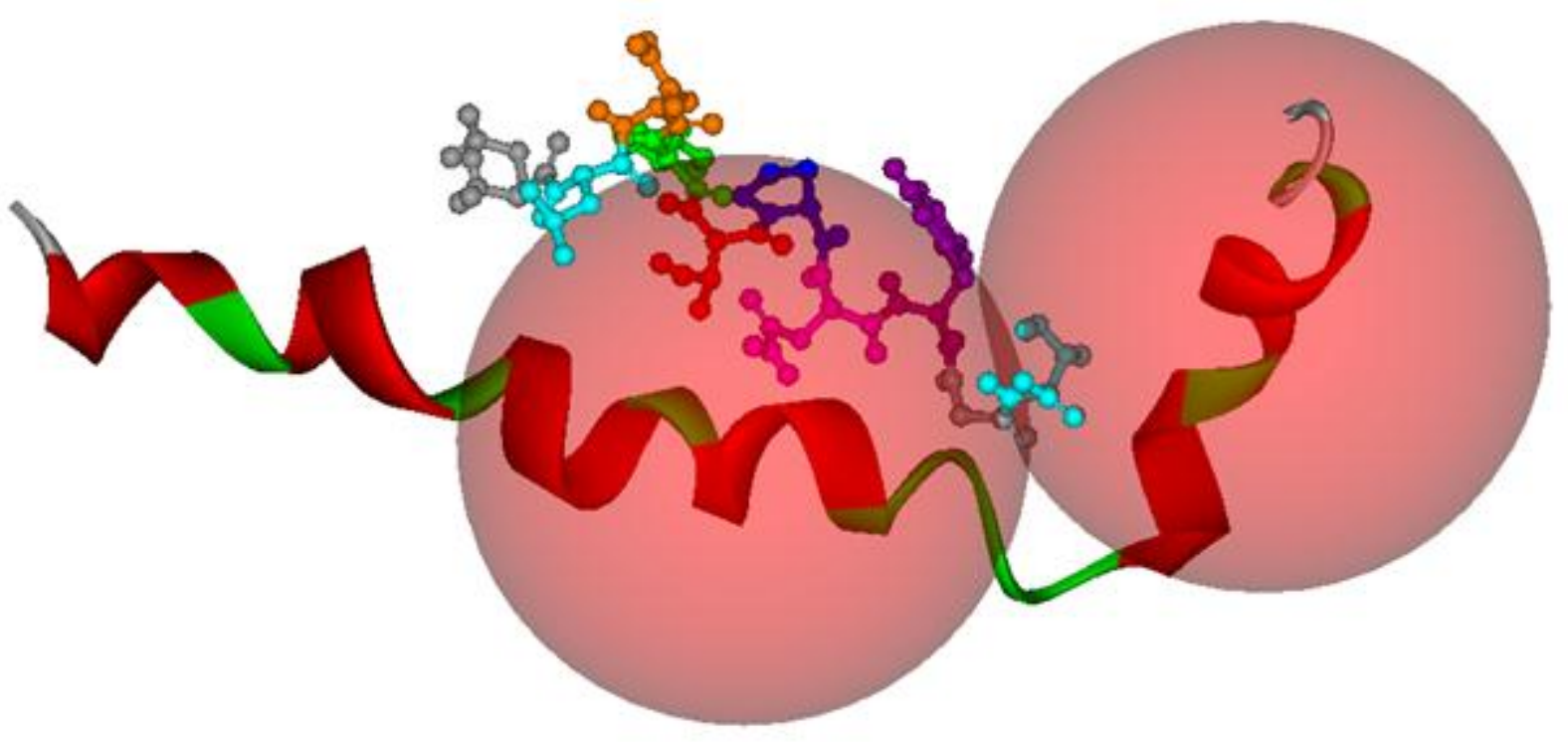

Figure 7:- ELNFTPNWGT with highest Z-Dock score (Pose 168) 


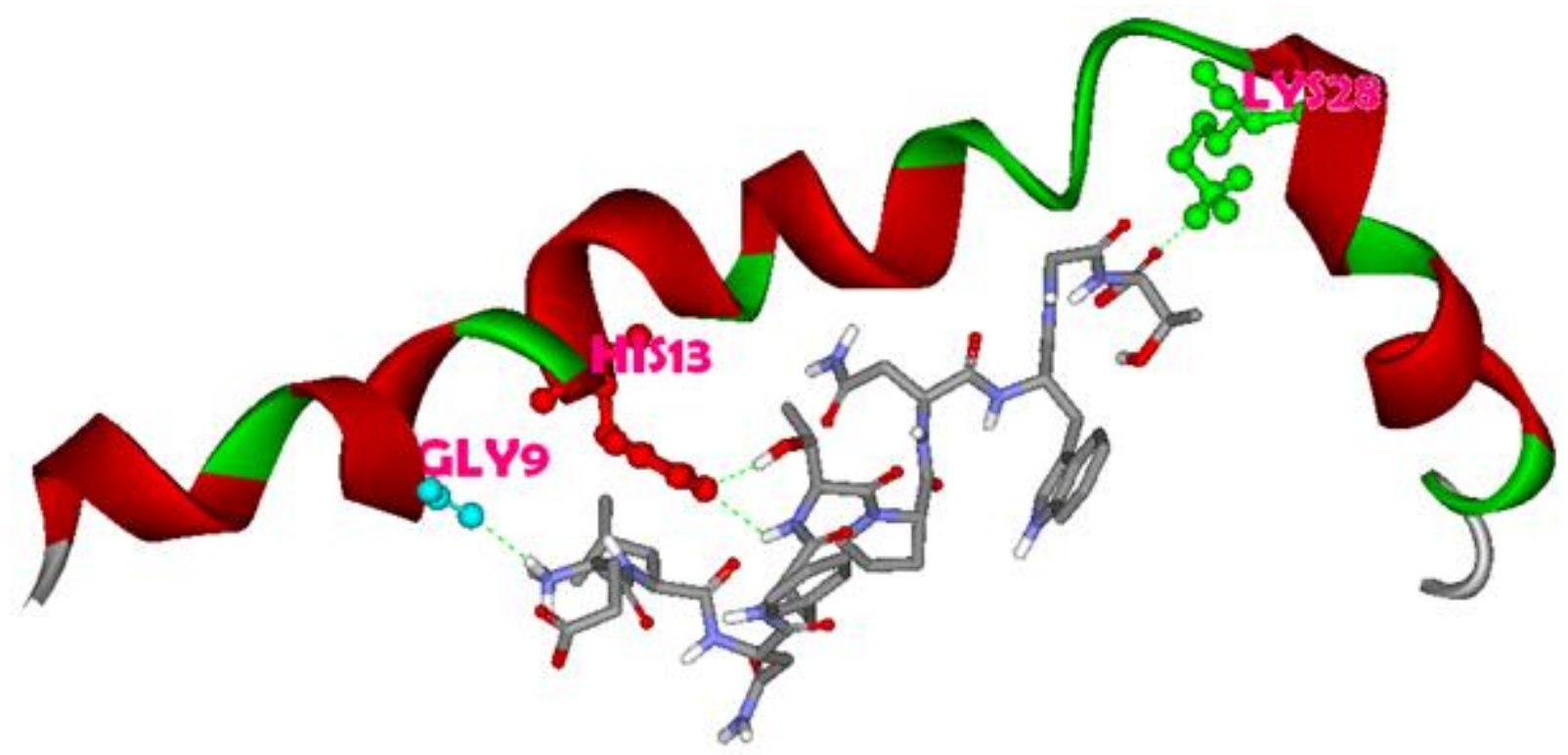

Figure 8:- ELNFTPNWGT with highest R-Dock score (Cluster 26)

\section{Discussions:-}

In this study, the main objective here was to target the major pathological lesions of Alzheimer's disease i.e., the Amyloid beta plaques with the help of Bioinformatics tools and design therapeutic molecules that would combat against these plaques and reduce their content in neurons which might result in an improved communication and thereby enhancing the condition of the diseased person.

Albeit, the current structure "PDB ID - 1IYT", culled as the query, is a resultant of NMR, it has been gleaned as the query as it was found to be the most paramount protein structure involved in the formation of Amyloid beta plaques, wherein the amyloidogenic regions were found to be around the regions in the length of $16-21$ amino acids and 32 - 36 amino acids around which the active site spheres were constructed. With the 42 small molecules or analogues (ref Introduction) of small molecules taken for performing protein - ligand docking studies with A $\beta$ minimized protein structure, the docking studies of the 42 small molecules with the amyloidogenic regions of the $A \beta$ gave no docked poses for the first active site viz., $16-21$ amyloidogenic region and gave 381 poses for the second active site viz., 32 - 36 amyloidogenic region among which the best ligand molecule was found to be Octadecylsulfate with the highest libdock score of 84.927.

Since the first objective had no significant effect on the first amyloidogenic region viz., 16 - 21 amino acid region, the second objective was consummated which showed a significant effect on both the amyloidogenic regions. Wherein, in the second objective, on the basis of Structure Activity Relationships (SAR) peptide sequences, similar to a physiological metalloproteinase named Neprilysin, were taken and subjected for construction of peptides which were later submitted for protein - peptide docking studies with the same amyloid beta protein structure which was used for the first objective by the aid of $\mathrm{Z}$ - Dock and $\mathrm{R}$ - Dock algorithms with the $16-21$ and $32-36$ amino acid regions as the active sites. By the whole of 10 best peptide sequences (ref Introduction) obtained by means of both protein blast and pepbank server the best peptide molecule was found to have the sequence ELNFTPNWGT which gave the highest z-dock score of 10.34 for the Pose 168 .

Although in silico studies represent a relatively new avenue of inquiry, it has begun to be used widely in studies which predict how drugs interact with the body and with pathogens. These in silico models exactly mimic the real models hence reducing lot of money and time. But as it stands that it still is an artificial environment quite different from that of living models, it is imperative that these molecules be tested under wet lab conditions to ensure the competence and efficacy of the conclusive data obtained from the bioinformatics work. 


\section{Acknowledgements:-}

It gives me immense pleasure to express my sincere regards and indebtedness to Mrs. P. Sahithi, Co-Author of the paper, Former Assistant Professor, University College of technology, Osmania University, Hyderabad, India, for her moral support, guidance and her valuable advices given to me during my work.

I would like to express my sincere gratitude to Mr. Enaganti Srinivas, Averin Biotech Institute, Hyderabad, India, for providing me with the requisite software tools that helped me in successfully finishing my work.

\section{References:-}

1. Charles G. Glabe et al, (2007). Small Molecule Inhibitors of Aggregation Indicate That Amyloid $\beta$ Oligomerization and Fibrillization Pathways Are Independent and Distinct. THE JOURNAL OF BIOLOGICAL CHEMISTRY. VOL. 282, NO. 14: 10311-10324.

2. Frank M. LaFerla, (2008). Amyloid- $\beta$ and tau in Alzheimer's disease. Nature Publishing Group.

3. Hardy, J.A., and Higgins, G.A. (1992). Alzheimer's disease: the amyloid cascade hypothesis. Science, Vol. 256: 184-185.

4. John Hardy et al, (2002). The Amyloid Hypothesis of Alzheimer's Disease: Progress and Problems on the Road to Therapeutics. SCIENCE, 297: 353 - 356.

5. Julie A. Carson and Anthony J. Turner (2002). $\beta$-Amyloid catabolism: roles for neprilysin (NEP) and other metallopeptidases? Journal of Neurochemistry, 81: 1-8.

6. N. N. Nalivaeva et al. (2012). The Alzheimer's Amyloid - Degrading Peptidase, Neprilysin: Can We Control It? International Journal of Alzheimer's Disease, doi:10.1155/2012/383796, 12 pages.

7. Nie Q et al, (2011). Small molecule inhibitors of amyloid $\beta$ peptide aggregation as a potential therapeutic strategy for Alzheimer's disease. Acta Pharmacologica Sinica, 32: 545-551. 